\title{
Impact of early versus delayed umbilical cord clamping on post partum blood loss: a randomized controlled trial
}

\author{
Yogiraj Vaijanathrao Chidre ${ }^{1 *}$, Vijayalakshmi Chirumamilla ${ }^{2}$
}

\author{
${ }^{1}$ Department of Obstetrics \& Gynaecology, MNR Medical College, Hyderabad, India \\ ${ }^{2}$ Department of Obstetrics \& Gynaecology, Medicity Institute of Medical Sciences, Hyderabad, India
}

Received: 01 June 2015

Accepted: 09 July 2015

\section{*Correspondence: \\ Dr. Yogiraj Vaijanathrao Chidre, \\ E-mail: cpmreddy@gmail.com}

Copyright: (C) the author(s), publisher and licensee Medip Academy. This is an open-access article distributed under the terms of the Creative Commons Attribution Non-Commercial License, which permits unrestricted non-commercial use, distribution, and reproduction in any medium, provided the original work is properly cited.

\begin{abstract}
Background: Evidence published to date has not clearly established the impact of the timing of cord clamping on postpartum blood loss and necessitates further research. The objective was to find out the impact of early and delayed cord clamping on the post partum blood loss and on infant haemoglobin and hematocrit at $90 \pm 7$ days postpartum.

Methods: A randomized, controlled trial was performed on 183 consenting women fulfilling eligibility criteria in labour ward of a teaching hospital in India. They were randomly assigned into early cord clamping or delayed cord clamping group; other components of active management of third stage of labour being same for all. The duration of third stage of labour was noted. Quantitative assessment of post partum blood loss was done using BRASSS-V drapes and weighing blood soaked pads. Cord blood haemoglobin and hematocrit at birth and infant haemoglobin and hematocrit at $90 \pm 7$ days were estimated. Statistical analysis done using students unpaired ' $t$ ' test.

Results: No difference was noted in the mean blood loss $(203.52 \pm 122.74 \mathrm{ml}$ versus $200.74 \pm 104.07 \mathrm{ml}$ in early and delayed cord clamping respectively) and the duration of third stage of labour (Mean: 296.59 \pm 98.97 seconds versus $281.79 \pm 104.59$ seconds for early and delayed clamping respectively). Infant haemoglobin at $90 \pm 7$ days was $11.07 \pm$ $1.27 \mathrm{gm} / \mathrm{dl}$ versus $12.70 \pm 1.41 \mathrm{gm} / \mathrm{dl}(\mathrm{p}=0.0000)$ and infant hematocrit at $90 \pm 7$ days was $34.13 \pm 3.93 \%$ versus 39.33 $\pm 4.88 \%$ in early and delayed clamping respectively $(\mathrm{p}=0.0000)$.

Conclusion: The timing of cord clamping has no impact on the mean blood loss and duration of third stage of labour. Delayed clamping significantly increases mean venous haemoglobin and hematocrit at $90 \pm 7$ days without increasing NICU admissions.
\end{abstract}

Keywords: Umbilical cord clamping, Delayed cord clamping, Early cord clamping, Post partum blood loss, Neonatal haemoglobin.

\section{INTRODUCTION}

The optimal timing of cord clamping has been a controversial issue for decades. No standard definitions of "early" or "late" cord clamping have been formulated. Policies for timing of cord clamping vary, from 60 seconds after birth, to greater than 60 seconds after the birth or when cord pulsation has ceased. ${ }^{1}$

Early cord clamping is part of active management of the third stage of labour which significantly decreases duration of third stage and postpartum blood loss. ${ }^{1-3}$
The disadvantages of early clamping include infant anaemia, increased incidence of acidemia, hypoxic ischemic brain damage, childhood mental disorders, increased risk of hypovolemia, iron loss, several blood disorders, type 2 diabetes and increase in the likelihood of feto-maternal transfusion as a larger volume of blood remains in the placenta. ${ }^{4-6}$

There is high prevalence of anaemia in India, 79.2\% children aged 6-35 months are anaemic. ${ }^{7}$ Infant anaemia is associated with increased mortality and impaired mental 
and motor development. The advantages of delayed cord clamping also include higher haemoglobin levels, additional iron stores, less fall in haemoglobin (by about 1 $\mathrm{g} / \mathrm{dl}$ ) at 3 months and less anaemia later in infancy. Delaying the clamping of the cord helps in early initiation and increased duration of breastfeeding after birth when the baby is in direct contact with the mother. ${ }^{5,8,9}$

Disadvantages of delayed clamping are increased risk of hypervolemia, hyper viscosity, polycythemia, respiratory symptoms and hyperbilirubinemia. According to a recent meta analysis, although late clamping is associated with a moderate increase in blood viscosity and increased rates of polycythemia, there is no evidence of any significant harm as measured by the need for phototherapy to treat jaundice or by admission to the NICU. A Cochrane review suggests delayed cord clamping increases the risk of jaundice requiring phototherapy. ${ }^{1,4,10,11}$

The International Confederation of Midwives and the International Federation of Gynecologists and Obstetricians have removed immediate cord clamping from their recommendations. ${ }^{12}$

Further research is needed to determine the minimum time required to provide maximum benefit of placental transfusion, advantages and disadvantages of cord clamping by comparing maternal and neonatal outcomes. Hence present study has been undertaken to study the impact of early and delayed cord clamping on the post partum blood loss, on duration of third stage of labour, neonatal morbidity and on infant haemoglobin and hematocrit at $90 \pm 7$ Days.

\section{METHODS:}

Source of data: All women with term, singleton uncomplicated pregnancies undergoing full term vaginal deliveries in labour room at MNR Medical college and Medicity institute of Medical sciences were invited to participate in the trial.

Study design: A hospital based prospective, partially blinded Randomized Controlled Trial (where the neonatal health care providers and laboratory personnel were unaware of the intervention used) was proposed.

Sample size: 106; 53 in each group.

According to a randomized controlled trial, blood loss was $373 \pm 366 \mathrm{ml}$ in early cord clamping group and $351 \pm 327 \mathrm{ml}$ had blood loss in delayed cord clamping group. Taking these as the two sample means, the level of significance as $5 \%$ and power of the test as $90 \%$; the sample size in early cord clamping and delayed cord clamping group was proposed as 53 in each of the two groups.

$$
\begin{aligned}
& \mathrm{x}_{1}{ }^{-}=373, \\
& \mathrm{x}_{2^{-}}=351
\end{aligned}
$$

$$
\begin{aligned}
& S_{1}=366 \\
& S_{2}=327
\end{aligned}
$$

Taking the level of significance as $5 \%(\alpha=0.05)$

$$
\mathrm{Z} \alpha=1.96
$$

Taking the power of the test as $90 \%(\beta=0.10)$

$$
\mathrm{Z} \beta=1.28
$$

The formula for calculating sample size is:

$$
\begin{gathered}
\mathrm{N}=2(\mathrm{Z} \alpha+\mathrm{Z} \beta)^{2} \mathrm{~S}^{2} \\
\left(\mathrm{x}_{1}{ }^{-}-\mathrm{x}_{2}{ }^{-}\right)^{2}
\end{gathered}
$$

With the above values, $\mathrm{N}=53$ in each group

However, as this was the minimum required size, to get higher effect size for the secondary outcomes also and for better result; larger sample size of 183 was taken.

\section{Selection criteria}

\section{Exclusion criteria included maternal conditions like}

Caesarean section (previous or planned in current pregnancy), Instrumental delivery, Anaemia (Hb less than $8 \mathrm{gm} / \mathrm{dl})$, Preterm labour, Known Medical /Surgical disorders, All other conditions including obstetric complications requiring early cord clamping (Rh sensitized pregnancy, Multiple pregnancy, Severe pre eclampsia/ eclampsia, Antepartum hemorrhage)

\section{Neonatal exclusion criteria included}

Need for neonatal resuscitation, Major congenital abnormalities, Tight nuchal cord necessitating early cord cutting, Fetal distress, Birth asphyxia

\section{Study protocol}

The study got ethical approval by Institutional Review Board of Jawaharlal Nehru Medical College, Belgaum, Karnataka, India. This was an academic study conducted in accordance with revised CONSORT Guidelines. ${ }^{13}$

Randomization: Assignment of the participants to the two groups was done using computer generated randomized number sequence list with block size of 2 into either early or delayed cord clamping groups. The randomization list was concealed and placed in opaque sealed envelopes. These envelopes were opened when a woman was in active labour and fulfilled the inclusion criteria.

Randomisation was done on admission to the labour ward, when the women were in the active first stage of 
labour. The eligibility status of the woman could change after initial screening but before randomization. Although study staff did not inform the women of their assignment, the nature of the intervention made it impossible to blind them. If an already randomized woman later became ineligible, the assigned allocation code was not re-used. The women were randomized into two groups: Early cord clamping group and Delayed cord clamping group.

Masking: Given the characteristics of the intervention, the obstetrician in charge of the intervention (umbilical cord clamping) could not be blinded. However, health professionals who made the neonatal evaluations after birth were not the ones present when infants were delivered and were not aware of the intervention used for the delivery. The personnel in charge of laboratory tests also were not aware of the intervention used.

Procedure: Informed written consent was taken after fulfilling eligibility criteria. The screening proforma was completed for every pregnant woman screened for enrolment to the study. A structured survey questionnaire was used to gather obstetrical and medical details of patients. Baseline maternal data with regard to age, medicinal iron intake, parity, socioeconomic status, detailed medical history was noted for all women. Detailed obstetric history was taken from all participants to recognize any high risk factors for PPH. Menstrual history was noted. A general physical examination and systemic examination including obstetric examination was done for all women. The diagnosis was noted. Delivery outcome of all eligible participants was monitored. Standard care was provided if women refused to participate in the study at any time. Baseline neonatal data with regard to birth weight, sex and gestation were recorded in all cases.

The subject ID assigned to the enrolled women identified all the data. After delivery all neonates were placed approximately $10 \mathrm{~cm}$ below the vaginal introitus between the legs of the mother, dried and wrapped in a warm towel. The neonates remained in this position until the cord was clamped. All other treatment including administration of 10 IU oxytocin after delivery of the baby and controlled cord traction for the delivery of placenta were similar for patients in both groups.

Infants were followed up till 3 months of age. No medicinal iron was given to any of these infants till the end of the study. The feeding patterns, weight gain, intervening morbidities like respiratory infections and diarrhoea during this period were carefully recorded.

Any mother or infant requiring treatments that deviated from the study protocol were excluded from the study.

Cord Clamping: Timing of cord clamping was noted using stopwatch. Early cord clamping was done within 30 seconds after child birth. Delayed cord clamping was done at 3 minutes or after cessation of cord pulsation, whichever occurred first. Third stage of labour was timed using stopwatch.

Measurement of blood loss: Quantitative assessment of post partum blood loss was done using BRASSS-V drapes and estimating weight of blood soaked gauzes and pads.

Uterine tone, measurement of blood loss, time of delivery of placenta, and other routine parameters were recorded by the health care provider. At birth, $2 \mathrm{ml}$ of cord blood was collected in EDTA containing vials for haemoglobin and hematocrit estimation. Secondary outcomes that were noted included possible side effects of delayed cord clamping in newborns including hyperbilirubinemia requiring phototherapy and need for NICU admissions. Infants were followed-up regularly until 3 months of age. $2 \mathrm{ml}$ of infant venous blood at $90 \pm 7$ days was collected in EDTA containing vials. Venous haemoglobin and hematocrit in the infant at $90 \pm 7$ days was measured in blood drawn from the antecubital vein.

Investigations: Estimation of cord blood haemoglobin and hematocrit (PCV) at birth was done using cyanmethaemoglobin method. Estimation of infant haemoglobin and venous hematocrit (PCV) at $90 \pm 7$ days was done using cyanmethaemoglobin method.

Data Analysis: Data were collected on standardised forms, and analysed with SPSS version 13.0 statistical software for Windows. Data analysis was performed using appropriate statistical tests.

\section{RESULTS}

Of the 716 women screened, 375 were randomized of which 183 were recruited in the study and included in the data analysis. 88 of the 183 women were randomized in the early cord clamping group and 95 in delayed cord clamping group. Of the 183 mother- infant pairs, 159 $(86.89 \%)$ completed the follow up at the third month whereas $24(13.11 \%)$ were lost to follow up.

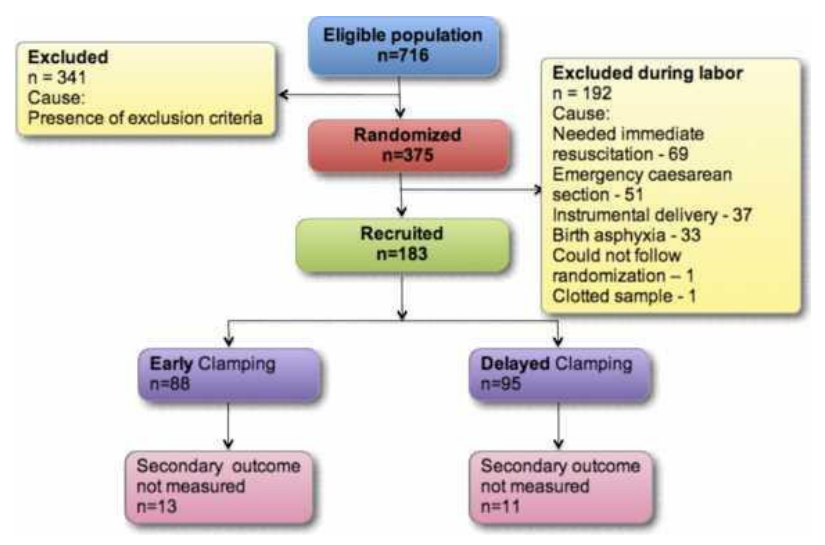

Figure 1: Flow chart of study population.

The groups were comparable for maternal demographic characteristics like age, religion, parity, education, 
antenatal care, medicinal iron intake and haemoglobin where no statistically significant difference was noted in the two groups. The systolic and diastolic blood pressure after delivery was also comparable in both groups. The infant's weight, Apgar score, gestation, sex and breastfeeding pattern at $90 \pm 7$ days, and infant haemoglobin and hematocrit values at birth were comparable with no significant difference between the two groups. Infant's haemoglobin and hematocrit values at $90 \pm 7$ days were significantly lower in early clamping group compared to those in the delayed clamping group. The time of cord clamping was $16.01 \pm 4.19 \mathrm{sec}$ in early cord clamping group and $101.98 \pm 16.59 \mathrm{sec}$ in delayed cord clamping group which was significantly different $(\mathrm{p}=0.0000)$.

Table 1: Mean blood loss during third stage of labour (in ml).

\begin{tabular}{|lll|}
\hline & $\begin{array}{l}\text { Early } \\
\text { clamping } \\
(\mathbf{N}=88)\end{array}$ & $\begin{array}{l}\text { Delayed } \\
\text { clamping } \\
(\mathbf{N}=95)\end{array}$ \\
& Mean+SD & Mean+SD \\
\hline Mean blood loss & $203.52 \pm 122.74$ & $200.74 \pm 104.07$ \\
\hline
\end{tabular}

No significant difference was found in the mean blood loss in early and delayed cord clamping groups $(\mathrm{p}=0.8683)$ in this study. (95\% CI, -30.32-35.88).

\section{Cord haemoglobin at birth}

At birth the cord haemoglobin in early clamping group was $15.33 \pm 1.65 \mathrm{gm} / \mathrm{dl}$ and in delayed cord clamp was $15.85 \pm 1.93 \mathrm{gm} / \mathrm{dl}$ which was not significantly different $(\mathrm{p}=0.0510)$.

\section{Cord hematocrit at birth}

At birth the cord hematocrit in early clamping group was $47.63 \% \pm 5.45 \%$ and in delayed cord clamp was $49.35 \% \pm$ $6.29 \%$ which was not significantly different $(\mathrm{p}=0.0501)$.

\section{NICU admissions after birth}

NICU admissions after birth were similar in both groups $(\mathrm{p}=0.4505)$. Of the $10 \mathrm{NICU}$ admissions, 7 admissions were for hyperbilirubinemia and 3 admissions for respiratory distress. None of the neonates were admitted in the NICU for birth asphyxia and they had transient tachypnoea which did not require any ventilatory support. Of the 5 NICU admissions in ECC, 3 admissions were for respiratory distress and 2 for hyperbilirubinemia. All the 5 NICU admissions in DCC were for hyperbilirubinemia.

\section{Neonatal hyper bilirubinemia requiring photo therapy}

There was no statistically significant difference $(p=0.1460)$ between the two groups for the parameter of neonatal hyperbilirubinemia requiring photo therapy.
The clinical course after discharge during the first month of life was similar in both groups, and at $90 \pm 7$ days no significant differences in relation to the infant's weight or frequency of exclusive breastfeeding were observed. One neonatal death was observed in the population under study due to respiratory illness after 1 month in the early cord clamping group.

Table 2: Infant venous haemoglobin and hematocrit at $90 \pm 7$ days in early and delayed groups.

\begin{tabular}{|lll|}
\hline & $\begin{array}{l}\text { Early clamping } \\
(\mathrm{N}=88) \\
\text { Mean+SD }\end{array}$ & $\begin{array}{l}\text { Delayed } \\
\text { clamping } \\
(\mathrm{N}=95) \\
\text { Mean+SD }\end{array}$ \\
\hline Hemoglobin & $\begin{array}{l}11.07 \pm 1.27 \\
\mathrm{gm} / \mathrm{dl}\end{array}$ & $\begin{array}{l}12.70 \pm 1.41 \\
\mathrm{gm} / \mathrm{dl}\end{array}$ \\
\hline Hematocrit & $34.13 \% \pm 3.93 \%$ & $39.33 \% \pm 4.88 \%$ \\
\hline
\end{tabular}

Infant haemoglobin at $90 \pm 7$ days in early cord clamp group was $11.07 \pm 1.27 \mathrm{gm} / \mathrm{dl}$ and in delayed cord clamp was $12.70 \pm 1.41 \mathrm{gm} / \mathrm{dl}$ and was significantly different. (95\% CI, 1.24-2.02). $\mathrm{P}=0.0000$ (HS). Infant hematocrit at $90 \pm 7$ days in early cord clamp group was $34.13 \% \pm$ $3.93 \%$ and in delayed cord clamp group was $39.33 \% \pm$ $4.88 \%$ and was significantly different. (95\% CI, 3.926.48). $\mathrm{P}=0.0000$ (HS) (Table 2).

\section{DISCUSSION}

This prospective randomized, controlled study evaluated the potential effects on the post partum blood loss and duration of third stage of labour, the haemoglobin and hematocrit status of the newborn and at 3 months at different cord-clamping time intervals.

\section{Mean blood loss}

In the present study, no significant difference $(\mathrm{p}=0.8683)$ was found in the mean blood loss in early and delayed cord clamping groups. Various studies have reported that late clamping is not associated with greater postpartum bleeding.

No differences were observed among the groups with respect to postpartum blood-loss volume, postpartum haemorrhage, and maternal hematocrit level 24 hours after birth in another RCT. However, this study included both vaginal and caesarean section birth as opposed to the other studies where only vaginal delivery outcome is assessed. The median maternal blood loss was $265 \mathrm{ml}$ in the early-cord-clamping group, $250 \mathrm{ml}$ in the 1-minute cord-clamping group, and $300 \mathrm{ml}$ in the 3 -minute cordclamping group. Postpartum hemorrhage (blood loss $>500$ $\mathrm{ml}$ ) was $26.8 \%$ in the early-cord-clamping group, $22.2 \%$ in the 1-minute cord-clamping group, and $25.4 \%$ in the 3 minute cord-clamping group. Severe postpartum hemorrhage (blood loss $>1000 \mathrm{ml}$ ) was $3.6 \%, 5.6 \%$, and $3.2 \%$ in each group, respectively. ${ }^{10}$ 
Maternal blood loss after delivery does not vary significantly between delayed cord clamping and immediate clamping groups in another study. ${ }^{5}$ The maternal blood loss after delivery did not vary significantly between delayed cord clamping and immediate clamping groups in these trials.

A Cochrane systematic review of 11 randomized trials including 2989 mother-infant pairs suggests that delaying clamping for at least 2-3 minutes seems not to increase the risk of postpartum haemorrhage which is in agreement with the results of the present study. ${ }^{1}$

\section{Mean Duration of third stage of labour}

No difference in the duration of third stage of labour was noted between the two groups in the present study. None of the published studies have compared the effect of early and delayed clamping on the duration of third stage of labour. However, an unpublished study by McDonald 1996, quoted in the Cochrane review concluded that instances of third stage greater than 30, nor 60 minutes, were not significantly different between the early and late cord clamping groups. ${ }^{1}$

\section{NICU admissions after birth}

There was no significant increase in the need for NICU admissions in the delayed cord clamping group in this study. In a meta analysis by Hutton et al, no significant differences in NICU admissions between late and early cord clamping (RR, 2.02; 95\% CI, 0.63 to 6.48 ) was noted. $^{4}$

\section{Neonatal hyperbilirubinemia requiring photo therapy}

In the present study, 02/88 (2.27\%) cases of neonatal hyperbilirubinemia requiring photo therapy were noted in early clamping group and 05/95 (5.26\%) in delayed cord clamping group which was not significantly different in the two groups $(\mathrm{p}=0.1460)$.

Cord clamping 1 to 3 minutes after delivery of the baby improves the iron status of the infant. Potential adverse effects of delayed cord clamping such as jaundice requiring phototherapy, should be considered. ${ }^{14}$

Few studies suggest that there may be an increased risk for hyperbilirubinemia, however phototherapy or exchange transfusion are not usually indicated. The incidence of hyperbilirubinemia requiring phototherapy was similar in both the groups, which goes along with other authors' observations. ${ }^{1,4,10,11}$

\section{Infant haemoglobin at $90 \pm 7$ days}

Infant haemoglobin at $90 \pm 7$ days in ECC group was $11.07 \pm 1.27 \mathrm{gm} / \mathrm{dl}$ and in DCC group was $12.70 \pm 1.41$ $\mathrm{gm} / \mathrm{dl}$ and was significantly different in the present study. A clinical trial from India by Geethanath et al found that the serum ferritin was higher but insignificant, in the delayed clamping group. The results of this study did not match with other trials. ${ }^{15}$ Gupta et al reported that the mean infant ferritin and $\mathrm{Hb}$ at 3 months were significantly higher in the DCC group than in the ECCC group. The mean decrease in $\mathrm{Hb}$ at 3 months adjusted for co-variates was significantly less in the DCC group. ${ }^{9}$

\section{Infant hematocrit at $90 \pm 7$ days}

Infant hematocrit at $90 \pm 7$ days in early cord clamp group was $34.13 \% \pm 3.93 \%$ and in delayed cord clamp group was $39.33 \% \pm 4.88 \%$ and was significantly different in the present study. (95\% CI, 3.92-6.48). $\mathrm{p}=0.0000$ (HS)

Similar results were also noted in other studies. $5,9,11$

Delayed cord clamping is compatible with active management of the third stage of labour. Uterotonic agents administered following birth and prior to cord clamping have been shown to increase the rate of placental transfusion and are thus likely to enhance the effect of delayed clamping. A joint statement from the International Federation of Gynaecology and Obstetrics and the International Confederation of Midwives on active management of the third stage of labor already recommends that delayed clamping be incorporated as part of the active management approach to placental delivery. In a recent literature review, similar practice recommendations pertaining to third-stage management were made for providing care in resource-poor settings. $1,16,4,17,14$

\section{Strengths and Limitations of the study}

Randomized design, partially blinded study, proper matching, and accurate measurement of blood loss. A limitation of this study is that mothers who were already assigned to a treatment group could later become ineligible. This could not be avoided in view of timing and nature of the intervention. In the present study, infant follow up was done only for $90 \pm 7$ days. However, the long term effects of the intervention (ECC and DCC) on the infants need to be evaluated with an extended follow up.

\section{CONCLUSION}

There is no significant difference between mean blood loss and duration of third stage of labour in early or delayed cord clamping groups. Neither significant differences nor harmful effects were observed among groups. Furthermore, this intervention seems to reduce the rate of neonatal anaemia. This practice has been shown to be safe and should be implemented to increase neonatal iron storage at birth.

Delayed cord clamping was associated with increased benefits to the infant (Higher $\mathrm{Hb} \%$ and hematocrit at $90 \quad \pm 7$ days) without increasing maternal (mean blood loss) or early neonatal morbidity or mortality (NICU admissions, hyperbilirubinemia requiring photo therapy). 
Delayed cord clamping is a simple, cost-free and safe delivery procedure that might offer a sustainable strategy to reduce early infant anaemia risk when other interventions are not yet feasible. It should be included in integrated programs aimed at reducing anaemia in young children in developing countries.

\section{Recommendations}

Delayed umbilical cord clamping practice should be implemented to reduce the incidence of neonatal anaemia in all term infants who have no indications for early cord clamping, especially in a resource poor country like India. The results of the present study are endorsed by the WHO Reproductive Health Library; World Health Organization, 2009. ${ }^{19}$

Funding: No funding sources

Conflict of interest: None declared

Ethical approval: The study was approved by the Institutional Ethics Committee

\section{REFERENCES}

1. McDonald SJ, Middleton P. Effect of timing of umbilical cord clamping of term infants on maternal and neonatal outcomes. Cochrane Database Syst Rev. 200816;(2):CD004074.

2. Winter C, Macfarlane A, Deneux-Tharaux C, Zhang $\mathrm{W}-\mathrm{H}$, Alexander S, Brocklehurst P, et al. Variations in policies for management of the third stage of labour and the immediate management of postpartum haemorrhage in Europe. BJOG. 2007;114:845-54.

3. Prendiville WJ, Elbourne D, McDonald S. Active versus expectant management in the third stage of labour. Cochrane Database of Systematic Reviews 2000, Issue 3.

4. Hutton EK, Hassan ES. Late vs. early clamping of the umbilical cord in full-term neonates: systematic review and meta-analysis of controlled trials. JAMA. 2007;297(11):1257-8.

5. Chaparro CM, Neufeld LM, Alavez GT, Cedillo REL, Dewey KG. Effect of timing of umbilical cord clamping on iron status in Mexican infants: a randomised controlled trial. Lancet. 2006;367:19972004.

6. N Wiberg, K Källén, P Olofsson. Delayed umbilical cord clamping at birth has effects on arterial and venous blood gases and lactate concentrations. BJOG. 2008;115(6):697-703.

7. 2005-2006 National Family Health Survey (NFHS-3) National Fact Sheet. Ministry of Health and Family Welfare Government of India.
8. Mercer JS. Current best evidence: a review of the literature on umbilical cord clamping. J Midwifery Womens Health. 2001;46:402-14.

9. Gupta R, Ramji S. Effect of delayed cord clamping on iron stores in infants born to anaemic mothers: a randomized controlled trial. Indian Pediatr. 2002;39 (2):130-5.

10. Ceriani Cernadas JM, Carroli G, Pellegrini L, Otano L, Ferreira M, Ricci C, et al. The Effect of Timing of Cord Clamping on Neonatal Venous Hematocrit Values and Clinical Outcome at Term: A Randomized, Controlled Trial. Pediatrics. 2006;117(4):779-86.

11. van Rheenen $\mathrm{P}$, Brabin BJ. Late umbilical cord clamping as an intervention for reducing iron deficiency in term infants in developing and industrialised countries: a systematic review. Ann Trop Paediatr. 2004;24(1):3-16.

12. Lalonde A, Daviss BA, Acosta A, Herschderfer K. Postpartum hemorrhage today: ICM/FIGO initiative 2004-2006. Int J Gynecol Obstet. 2006;94:243-53.

13. Altman DG, Schulz KF, Moher D, Egger M, Davidoff F, Elbourne D, et al. The revised CONSORT statement for reporting randomized trials: explanation and elaboration. Annals of Internal Medicine. 2001;134:663-94.

14. Abalos E. Effect of timing of umbilical cord clamping of term infants on maternal and neonatal outcomes: RHL commentary. The WHO Reproductive Health Library; Geneva: World Health Organization, 2009.

15. Geethanath RM, Ramji S, Thirupuram S, Rao YN. Effects of timing of cord clamping on the iron stores of infants at 3 months. Indian Pediatr. 1997;34:1036.

16. Versaevel. N, Darling L. Prevention and Management of Postpartum Haemorrhage. AOM Clinical Practice Guideline, No. 9, 2006 Mar. Available from: http://www.aom.on.ca/files/ Office_Documents/Communications/No_9_ Prevention_and_Management_of_PPH.doc.

17. WHO recommendations for the prevention of postpartum haemorrhage. WHO. Department of Making Pregnancy Safer. Geneva: WHO, 2007.

Cite this article as: Chidre YV, Chirumamilla V. Impact of early versus delayed umbilical cord clamping on post partum blood loss: a randomized controlled trial. Int J Reprod Contracept Obstet Gynecol 2015;4:1103-8. 